\title{
LOS PERFILES PROFESIONALES DE LA COMUNICACIÓN EN LOS GRADOS DE DE PUBLICIDADY RELACIONES PÚBLICAS. EL CASO DE LA UNIVERSIDADE DE VIGO
}

\author{
Montse Vázquez-Gestal ${ }^{1}$. Universidad de de Vigo. España \\ mvgestal@uvigo.es
}

\section{RESUMEN}

El progreso tecnológico ha provocado innumerables cambios en nuestra percepción del mundo, en nuestra manera de relacionarnos, en el ámbito laboral y, como no podía ser de otra manera, en los trabajos comunicativos. Con esta perspectiva hemos querido analizar esta situación en un contexto geográfico determinado para saber si en el caso de Galicia, y más concretamente la facultad de Ciencias Sociales y de la Comunicación de la Universidade de Vigo, ha introducido en sus estudios de Publicidad y Relaciones Públicas las necesidades que hoy se exigen y se van a exigir en un futuro al trabajador de la comunicación, cuya labor debe asentarse en un panorama tan cambiante y rápido que apenas uno puede percatarse de lo que sucede y de las innovaciones que aparecen, un mundo en el que cuando añadimos, comprendemos y manejamos una nueva aplicación en nuestro móvil ésta ya ha sido superada por la versión siguiente. El artículo pretende analizar el título de Grado en Publicidad y Relaciones Públicas puesto en marcha por la Universidade de Vigo con la intención de conocer si éste responde a las necesidades reales que plantean los profesionales y la sociedad.

PALABRAS CLAVE: Formación - Publicidad - Relaciones públicas - Grados

\footnotetext{
${ }^{1}$ Autor Correspondiente

Montse Vázquez Gestal. Profesora Titular de Universidade del área de Comunicación Audiovisual y Publicidad. Facultad de Ciencias Sociales y de la Comunicación. Universidade de Vigo. España.

Correo: mvgestal@uvigo.es
} 


\title{
PROFESSIONAL PROFILES IN COMMUNICATION AS DESIGNED IN THE BACHELOR'S DEGREE IN ADVERTISING AND PUBLIC RELATIONS: THE UNIVERSIDAD DE VIGO CASE STUDY.
}

\begin{abstract}
Technological progress has produced many changes in our perception of the world, interpersonal relationships, work environment and, more specifically, in the communication sphere. Taking into consideration this process, we aim to analyse how the Faculty of Social Sciences and Communication of the Universidad de Vigo is dealing with these phenomena when designing and reforming the core curriculum of its Bachelor's Degree in Advertising and Public Relationships, which has to train future professionals in communication in a world of permanent media technology conversion. Through this specific case, the ultimate goal of this article is to assess how this degree's course of study meets the professional requirements which are presently demanded by the job market.
\end{abstract}

KEYWORDS: Training - Advertising - Public Relations - Degrees

\section{INTRODUCCIÓN}

El progreso tecnológico ocurrido en las últimas décadas ha provocado innumerables cambios en nuestra percepción del mundo y en nuestra manera de relacionarnos, pero no solo eso, la revolución tecnológica ha resultado una revolución en el ámbito laboral, en cualquiera de ellos y, como no podía ser de otra manera, de una forma explosiva en los trabajos comunicativos. Estos cambios deberían aplicarse también a la formación que los comunicadores, dentro de la rama elegida (periodismo, publicidad, relaciones públicas o comunicación audiovisual, como se han conocido tradicionalmente en las facultades) deberían recibir en las facultades donde estos estudios se imparten.

Acabamos de iniciar una profunda remodelación de los estudios universitarios de la mano del denominado plan Bolonia o el EEES, pero deberíamos analizar si estos nuevos cambios en los planes de estudio han escuchado a un entorno cambiante y se han apresurado a introducir los conocimientos, habilidades y destrezas que el mundo laboral exige en el ámbito de la comunicación y que la reforma europea no ha parado de solicitar. 
Con esta perspectiva hemos querido analizar esta situación en un contexto geográfico determinado para saber si en el caso de Galicia, y más concretamente la facultad de Ciencias Sociales y de la Comunicación de la Universidad de de Vigo, ha introducido en sus estudios de Publicidad y Relaciones Públicas las necesidades que hoy se exigen y se van a exigir en un futuro al trabajador de la comunicación, cuya labor debe asentarse en un panorama tan cambiante y rápido que apenas uno puede percatarse de lo que sucede y de las innovaciones que aparecen, un mundo en el que cuando añadimos, comprendemos y manejamos una nueva aplicación en nuestro móvil ésta ya ha sido superada por la versión siguiente.

En primer lugar es nuestra intención señalar algunas necesidades $\mathrm{u}$ opiniones que, sobre el futuro desarrollo de la publicidad y las relaciones públicas, hemos recogido en distintos artículos de publicitarios actuales para conocer lo que se les va a pedir a los futuros profesionales de la comunicación y comprobar si se han tenido en cuenta esas necesidades y se han visto reflejadas en las nuevas titulaciones, si se ha aprovechado el cambio para incluir las necesidades reales de estas profesiones, para convertir el aprendizaje en una oportunidad de futuro o si, por el contrario, el Plan Bolonia ha sido una oportunidad perdida para terminar con la tan manida frase de que la universidad está de espaldas al mundo real.

\section{METODOLOGÍA}

La presente investigación tiene su sustento metodológico en el método analíticosintético, el cual facilita el análisis el título de Grado en Publicidad y Relaciones Públicas puesto en marcha por la Universidad de de Vigo para así valorar y conocer sus particularidades, y simultáneamente a través de la síntesis, conocer si éste programa de estudio responde a las necesidades reales que plantean los profesionales y la sociedad.

\section{ANÁLISIS Y DISCUSIÓN}

\subsection{La revolución tecnológica y la Comunicación}

Hay muy poco nuevo que contar acerca de lo que Internet y el desarrollo de las nuevas tecnologías ha significado para el mundo actual, la simplificación de muchos trabajos, el ahorro de costes obtenido por la cantidad de actividades y transacciones on line que se desarrollan, el progreso en comodidad y seguridad o el aumento de las posibilidades de ocio a través de una amplio abanico de posibilidades.

Tampoco vamos a descubrir las posibilidades abiertas en el mundo de la 
productos audiovisuales, sin dejar de mencionar la aparición de nuevos canales o soportes comunicativos que han ido desplazando a los medios tradicionales y que forman parte ya de nuestro ámbito más cotidiano, sino que también hay que hacer hincapié en la aparición de nuevos modelos de trabajo, nuevas figuras y nuevos roles asociados en muchos casos a la propia evolución de cada actividad comunicativa.

Y ante esta situación cabe preguntarnos si los centros de formación han tomado nota de este entorno versátil para intentar cambiar, mejorar, ampliar, reestructurar, actualizar, completar... todos los conocimientos que los estudiantes necesitan para convertirse en unos profesionales más adaptados a las circunstancias que les han tocado vivir, pero que también serán distintas a las que se encontrarán cuando terminen su período académico. Se trata de replantearse ciertos tipos de conocimientos para mejorar la preparación del estudiante sin olvidar, evidentemente, qué tipo de centros son las facultades y qué tipo de enseñanzas deben impartirse en ellas.

Al margen de las necesidades básicas que todos conocemos y que siguen existiendo en prácticamente todos los estudios de comunicación, la evolución del entorno nos lleva a plantearnos que necesitarán conocer los alumnos cuando salgan a un mercado laboral tan cambiante. Bien es cierto que nunca hubo tal cantidad de información a nuestro alcance y tan poco tiempo para estar al día de todo lo que ocurre. Los medios ya no son lo que eran y no se trata solo de conocer "lo de siempre" sino de conocer lo que todavía no existe.

\subsection{La formación de los nuevos profesionales}

El consumidor se ha convertido en alguien tremendamente exigente que en ocasiones va por delante del producto o de la marca, un individuo conocedor y creador de contenidos que ya no espera sino que busca lo que le interesa. Internet ha cambiado toda la perspectiva del mundo y, por tanto también de la publicidad y de las relaciones públicas, ¿preparamos a nuestros alumnos para ello? $\mathrm{O}$ tal vez cabría preguntarnos, ¿estamos nosotros preparados para ello? El nuevo medio lo ha modificado absolutamente todo y él da las pautas de lo que será necesario en la comunicación publicitaria del futuro.

Las nuevas relaciones del consumidor con las marcas o a la inversa, marcan el futuro publicitario, hablamos de experiencias, de conversaciones, el protagonista más que nunca es el cliente, ese cliente que no solo consume sino que construye, que no solo compra en internet sino que se informa, se entretiene, incluye contenidos, crea su espacio...hablamos de una nueva comunicación con un: 
Realmente el cliente es el rey y, sobre todo, porque él se ha dado cuenta de ello, él conoce, él busca, él critica, hoy hay que conocerle mejor que nunca, con él hay que conversar y a él hay que divertir cuando le presentamos un nuevo producto o un nuevo servicio, porque no solo competimos con otros productos para conseguir su atención, en la red competimos con todo, videojuegos, viajes, deportes, contactos...

El tiempo del consumidor es limitado y debemos conseguir que quiera vernos así que debemos entretenerle, mostrarle algo que valga la pena ver, compartir. Por ello la publicidad debe seducir más que nunca, implicar, entretener, aparece un nuevo concepto denominado Advertainment, o lo que García denomina nueva publicidad, aquella que crea ideas para llevar al consumidor a la marca, se trata de "crear ideas que la gente quiera incluir en su tiempo" (García, 2008, p. 10).

El conocimiento del consumidor se sitúa otra vez como el pilar central que los profesionales o futuros profesionales deben conocer y eso significa observarlo desde distintas perspectivas no solo comunicativas sino también sociológicas, psicológicas, empresariales o culturales para poder conocer tendencias, anticiparse o no retrasarse a la hora de ofrecerle nuevos contenidos y experiencias, el consumidor interviene realmente en la comunicación (Bravo Navalpotro, 2010), la tecnología lo permite y el anunciante debe saber utilizarla para ganar su atención y vender, ya que ese sigue siendo el objetivo fundamental de esta actividad.

\subsection{La importancia de las ideas}

Pero además de la importancia sobre manera de este nuevo consumidor/cliente/usuario/prosumer hay que plantearse una nueva forma de hacer las cosas, internet ha traído nuevos formatos, tipos, mensajes, códigos.... Sin embargo, parece que vuelve a destacarse de nuevo la importancia de algo tan simple y sencillo que en ocasiones hasta se ha olvidado y eso es la idea.

Las ideas siguen sido lo importante en este nuevo sistema comunicativo y saber cómo, dónde y cuándo encontrarlas se plantea de nuevo como una panacea a la que no puede renunciarse, porque la búsqueda de nuevas ideas es lo que ha movido al mundo siempre y en esta nueva revolución digital tampoco es una excepción, hablamos de ideas y no de herramientas ni de tecnología.

Las ideas nos sacaron de las cavernas, nos hicieron llegar a la luna y nos enseñan que mamá tiene superpoderes ya que enciende y apaga con magia la nueva plancha Bosch. En este momento de ritmo frenético, donde uno no sabe a donde mirar o que fórmula publicitaria elegir, la creatividad es la única respuesta, la idea es la solución 
El tiempo parece que corre en contra del comunicador pero es preciso no perder la perspectiva, se trata de vender, las ideas siempre han funcionado y seguirán haciéndolo ahora, de tal manera que saber producirlas y buscarlas seguirá siendo algo imprescindible, "lo importante por encima de todo son las ideas. Sepamos hacer que salgan, sepamos identificarlas y sepamos manejarlas para que se conviertan en realidad y hagan funcionar nuestros negocios. Las ideas son lo único que ni antes eran analógico ni en el futuro va a ser digital" (Muñoz, 2010).

Recordemos la importancia de las ideas con un hecho nuevo promovido desde una entidad como el Club de Creativos quien ha establecido que, a partir de esta edición, en la construcción de su Anuario las piezas elegidas lo serán en función de las ideas y no siguiendo una categorización apoyada en el medio elegido. Ideas y ejecución ${ }^{2}$, eso es lo importante en la construcción comunicativa actual.

Podríamos decir que hay grandes bloques de conocimientos importantes para estos nuevos comunicadores, por un lado el mundo del consumidor desde todas sus perspectivas. Conocer como actúa, se informa, se entretiene, se relaciona, como piensa, sus necesidades, sus miedos, sus anhelos... vuelve a ser el centro de toda esta actividad comunicativa, como lo ha sido siempre, pero ahora de una forma mucho más auténtica pues la tecnología ha permitido que sea él realmente quien mande y quién marque los tiempos en esta actividad. Eso significa que el ser humano y todas sus variables se vuelven imprescindibles en la formación de un profesional.

Por otro lado, todo lo que se refiere a las ideas en el sentido no solo de ideas en la elaboración de mensajes, porque la creatividad es necesaria para encontrar nuevos soportes, formatos o maneras de entrar en contacto entre anunciante y cliente, las redes sociales son solo una pequeña parte de lo que será el futuro, como lo fueron las primera páginas web, o la radio la tv y la prensa en su día. Medios que no desaparecen sino que deben seguir conociéndose y reinventándose porque siguen siendo necesarios en la construcción comunicativa actual.

$\mathrm{Y}$ en el conocimiento de los medios debe incluirse internet y todas las herramientas que de él han derivado, pues conocerlas nos llevara a mejores fórmulas, de la misma manera que el estudio de los medios tradicionales nos llevó a construir mejores mensajes, mejores formatos, mejores programas... No se trata de conocer programas de diseños o las redes sociales, se trata de conocer su conexión al individuo, sus funciones, sus posibilidades y usos más allá de saber manejar un programa.

\footnotetext{
${ }^{2}$ El Anuario del S. XXI. El Club de Creativos ha decidido cambiar su forma de construir el Anuario
} 
Pero además habría de mencionarse otro elemento más, hablamos de una titulación en Publicidad y Relaciones Públicas, somos conscientes que todo lo dicho anteriormente no solo se aplica al campo publicitario sino también a la disciplina de las Relaciones Públicas, de la que solemos olvidarnos, y que se ve reforzada en la actualidad por esas exigencias de los consumidores hacia empresas, anunciantes, administraciones... Son un campo de actuación cada vez más destacado, afortunadamente, y donde también surgen nuevas formas y fórmulas. Las responsabilidades de todo tipo exigidas por parte de los individuos se ha convertido en un campo de abono perfecto para las Relaciones Públicas desde ámbitos que hasta hace muy pocos años casi eran prácticamente desconocido (Matatia y Saltzman, 2001).

Planteadas estas posibilidades y oportunidades que se desgajan de la opinión de los profesionales, veamos si éstas se recogen en las nuevas titulaciones.

\subsection{Qué busca Bolonia}

La idea de una Europa unida es algo que forma parte ya de nuestra propia cotidianeidad y que hemos ido viviendo a lo largo de las últimas décadas, un proceso político y económico sobretodo pero que ha querido también desarrollarse al amparo de dimensiones científicas, intelectuales, tecnológicas y culturales. En este sentido se enmarca el desarrollo del denominado Espacio Europeo de Educación Superior ${ }^{3}$, nacido en la Declaración de Sorbona del 25 de mayo de 1998 firmada por los ministros de educación de Francia, Alemania, Italia y Reino Unido y concebida como un primer paso para el cambio de la enseñanza superior en Europa.

La Declaración de Bolonia del año siguiente firmada ya por 30 países sienta las bases para el cambio apoyándose en los principios de calidad, movilidad, diversidad y competitividad; pero además busca obtener dos objetivos estratégicos: el incremento de empleo en la UE y cómo convertir Europa en un polo de atracción para estudiantes y profesores de otras partes del mundo con este nuevo sistema. Y así comenzó el proceso de cambio en Europa y España, con la intención de que al final de 2010 las nuevas enseñanzas de grados, según la denominación europea, estuvieran iniciadas en el ámbito académico español con la creación de los nuevos grados. En el caso de la Comunicación, en torno a las tres titulaciones tradicionales: Periodismo, Publicidad y Relaciones Públicas y Comunicación Audiovisual, todas de cuatro años y todas presentes en la comunidad gallega, la primera de ellas en Santiago de Compostela, la segunda en el campus de Pontevedra de la Universidade de Vigo y la tercera titulación repetida en las tres universidades: Santiago, Vigo y Coruña. 
Desde los inicios de este milenio, el EEES y todo lo referido al Plan Bolonia ha estado presente en la vida universitaria con una fuerza importante, ya que comenzaba la verdadera etapa de trabajo a la que se enfrentaba la Universidad, un proceso que mencionaba Docampo Amoedo en su toma de posesión como Rector de la Universidade de Vigo en el año 2002 al señalar que entre los retos de la universidad se encontraban "la conformación del espacio europeo de la investigación y de la educación superior; la progresiva introducción de las tecnologías de la información y las comunicaciones en la investigación, en la docencia y en la gestión; el cambio de paradigma educativo, que ha de asociarse al aprendizaje permanente; la mayor interrelación entre la universidad y la sociedad, y el nuevo marco legislativo abierto por la LOU (...), la europeización pasa hoy a formar parte de la visión estratégica de las universidades, retomando el concepto renacentista de universidad abierta, permeable a las ideas que aportan estudiantes y profesores que se mueven en libertad entre las instituciones educativas de diferentes países" (www.universia.es).

El Libro Blanco de Comunicación fue aprobado en el año 2005, allí se recogieron las conclusiones de las tres Comisiones, una por titulación, bajo la supervisión de un único grupo de trabajo. Tras su aprobación comienzan los trabajos en las distintas universidades, entre ellas, evidentemente, las gallegas, lo que significa en las distintas facultades la creación de comisiones y grupos que desarrollaron los futuros estudios adaptados a las nuevas disposiciones.

\subsection{El grado de Publicidad y Relaciones Públicas en la UVigo}

Esta titulación de imparte de manera única en la Universidad de Vigo, concretamente en la Facultad de Ciencias Sociales y de la Comunicación en su campus de Pontevedra desde el año 1994. Tras pasar por dos planes de estudio, en el curso 20092010 se iniciaron los estudios de grado.

A lo largo de todo el 2008 se desarrollaron los trabajos de la comisión elegida a tal efecto en la Junta de Facultad de la facultad de Ciencias Sociales y de la Comunicación ${ }^{4}$. Su primera reunión se celebró en marzo de 2008, la propuesta para el nuevo plan de estudios de grado se finalizó el 17 de julio, tras el período de alegaciones fue remitida a la Comisión de Organización Académica y Profesorado de la Universidade de Vigo el 1 de octubre y aprobada en el Consello de Gobierno de la Uvigo en noviembre de 2008. Tras ello comenzó su introducción en el programa Verifica y continuó con sus trámites correspondientes hasta su publicación en el BOE el 1 de noviembre de 2010. 
En la memoria realizada se establecen, al amparo de los establecidos en el Libro Blanco de Títulos de Grado en Comunicación, los objetivos de la titulación de Publicidad y Relaciones Públicas, tres generales (enfocados a dotar al estudiante de la formación e instrumentos necesarios para el correcto desarrollo de la profesión, su preparación para la práctica profesional, y su capacitación para poder continuar su formación a través de los posgrados) y un total de trece específicos que los estudiantes precisan adquirir para poder desarrollar los objetivos generales

a) Adquirir conocimientos tanto técnicos como humanísticos, desde un punto de vista global e internacional y atendiendo a las necesidades propias del entorno, para obtener una competencia contextual básica de orden transdisciplinar que contribuya a una adecuada preparación profesional.

b) Adquirir un conocimiento exhaustivo de los fundamentos teóricos y prácticos que conforman el fenómeno de la comunicación, especialmente la publicitaria y de las relaciones públicas, su función en la sociedad moderna y sus metodologías y técnicas de investigación, para su aplicación a la realidad de las organizaciones o en el ámbito académico e investigador.

c) Adquirir conocimientos sobre el panorama actual de la labor del profesional de la comunicación, especialmente publicitaria y de las relaciones públicas, así como sobre el contexto social, cultural, político, económico y empresarial en el que se inserta su trabajo para adecuar, de forma responsable, su labor al mismo.

d) Adquirir conocimientos sobre el diseño y creación de políticas y estrategias comunicativas con el fin de desarrollar la capacidad de identificar, gestionar y atender las necesidades propias de las empresas, tanto desde la perspectiva del departamento de comunicación del anunciante como desde la empresa de comunicación.

e) Adquirir conocimientos sobre las capacidades expresivas y particularidades de cada uno de los medios, soportes y formatos publicitarios para la elaboración de mensajes y campañas de comunicación, desarrollando la habilidad para analizar y seleccionar los vehículos de difusión de acuerdo con las estrategias de comunicación (tanto en medios convencionales como en medios no convencionales).

f) Adquirir conocimientos teóricos y prácticos sobre las tecnologías de la comunicación y sobre el desarrollo y posibilidades de los nuevos soportes, para su aplicación eficaz en el ámbito de la publicidad y las relaciones públicas.

g) Adauirir conocimientos sobre los procedimientos de trabaio 
h) Desarrollar la capacidad para implementar estrategias de comunicación coherentes, por medio de una creatividad eficaz, midiendo posteriormente sus resultados y extrayendo las conclusiones oportunas.

i) Desarrollar la capacidad para construir, planificar y evaluar acciones y discursos propios de la comunicación publicitaria y de las relaciones públicas para la elaboración de campañas de comunicación, adecuando las estrategias y los mensajes a los objetivos de comunicación y a los diferentes públicos.

j) Desarrollar la capacidad analítica, crítica e interpretativa, así como la capacidad desarrollar de nuevos enfoques creativos en las tareas asignadas en el ámbito profesional, siempre con criterios de adaptación al entorno y de innovación continua.

k) Desarrollar la capacidad de ejercer el quehacer comunicativo con una conducta ética, tanto a través del conocimiento teórico-práctico de los códigos deontológicos de la publicidad vigentes y de la autorregulación de la profesión como del desarrollo de una conciencia de responsabilidad social y cultural.

1) Desarrollar la capacidad de comunicarse y expresarse eficazmente y con coherencia en su ejercicio profesional.

m) Adquirir una actitud estratégica que permita la anticipación y adaptación a los cambios, la detección y resolución de problemas, el desarrollo y optimización de sinergias grupales y la toma de decisiones.

\subsection{Los nuevos perfiles profesionales y su desarrollo}

Teniendo en cuenta dichos objetivos se establecen los perfiles profesionales que derivan del nuevo título:

a) Gestores y estrategas publicitarios: profesionales especializados en la puesta en marcha del proceso de planificación estratégica desde la relación con el cliente- anunciante hasta su evaluación final mediante el control de la eficacia. Coordinan los distintos pasos de este proceso desarrollando proyectos de investigación publicitaria y tomando decisiones en la planificación de medios. En este campo, determinan la idoneidad y características de los soportes para la difusión de campañas de comunicación, así como el control y seguimiento de la audiencia de dichos medios y su eficacia. Desarrollará su actividad en empresas de comunicación en sentido amplio (agencias de publicidad, consultoras y debartamentos de comunicación). 
b) Creativos: profesionales conocedores de todo el proceso creativo publicitario, que abarca los procesos relacionados con el briefing, su síntesis en una idea creativa, la conceptualización en herramientas y mensajes publicitarios, la realización y elaboración de productos impresos o audiovisuales y las diferentes técnicas de presentación del arte final. Así mismo, trabajará en la conversión simbólica y gráfica de los conceptos de identidad corporativa. Para ello se identifican especialidades como Creativo, Director Creativo, Diseñador gráfico (con una doble posibilidad en producción gráfica y producción audiovisual), Director de Arte, Redactor Publicitario y Productor Publicitario en Nuevas Tecnologías.

c) Directores de comunicación y relaciones públicas: profesionales que ejercen su actividad en el ámbito de las organizaciones (públicas, privadas o del Tercer Sector) desarrollando las siguientes funciones: gestión estratégica de las relaciones con los públicos internos y externos, así como de la imagen corporativa y la reputación; elaboración, gestión y control de los planes de comunicación; definición de estrategias y tácticas de comunicación corporativa, institucional o política, planificación y desarrollo de programas y campañas de relaciones públicas. Técnicos de comunicación y relaciones públicas que aplican, desarrollan y ejecutan acciones comunicativas y de relaciones públicas en el marco de un programa o campaña concreta, empleando las herramientas específicas de interrelación y comunicación interna y externa. Profesionales que ejercen sus actividades directivas y técnicas en consultoras de comunicación y relaciones públicas. Responsables de protocolo.

Llama la atención la separación de perfiles en torno a las actividades publicitarias y de relaciones públicas de una manera tan marcada, teniendo en cuenta que cada vez se tocan más las distintas actividades comunicativas en cuanto a la elaboración de trabajos. Así como también llama la atención la ausencia de nuevos roles profesionales, desarrollando simplemente los tres más habituales.

La presencia de estos perfiles y la preparación de los alumnos para su desarrollo configuran los contenidos en las distintas materias que se impartirán en la titulación y que se repartirán en tres módulos:

a) Fundamentos y contexto de la comunicación: 66 créditos ECTS repartidos en un total de 11 materias de las áreas de Periodismo (4), Ciencia Política (0.5), Estadística (0.5), Etica (1), Sociología (1), Organización de Empresas y 
Se trata de materias que deberían ayudar al alumno a situarse en el entorno profesional que le tocará vivir y a conocer cuestiones básicas de áreas cercanas a su trabajo y que deberían darle las pautas para interpretar los cambios desde el punto de vista político, ético, empresarial, comunicativo... Hay una disminución de contenidos más transversales con respecto a los planes de estudios anteriores donde existían materias de arte, historia, sistemas políticos, idiomas... que ahora desaparecen.

La importancia de contenidos no tan "actuales" es, sin embargo, algo destacado por profesionales del sector, podemos señalar las palabras del publicitario Santiago Romero, a su paso precisamente por esta facultad donde destacó "la importancia de adquirir conocimientos 'atemporales', ir desarrollando habilidades afines a la personalidad de cada uno, en las que después poder especializarse, dejando a un lado la tecnología, que cambia constantemente. 'Me da igual que escribas Hamlet a máquina o a lápiz, lo que importa es que escribas Hamlet', así ejemplifica la importancia de trabajar las capacidades necesarias para la creación audiovisual. En definitiva, en el mundo publicitario y audiovisual se búscan 'personas que sean capaces de aprender, con ganas y que estén dispuestas a trabajar'" (www.duvi.es).

Ese tipo de conocimientos, que ayudan a encontrar referencias conocidas para el consumidor en muchas ocasiones y que nos ayudan a conectar con él, han perdido peso en el nuevo grado.

a) Módulo de Comunicación Publicitaria: 120 créditos ECTS. Es el que incluye el mayor número de materias y el mayor de los módulos con diferencia ya que, como veremos posteriormente el módulo de Relaciones Públicas es de 66 créditos ECTS. En total 20 materias, de las que 14 serían obligatorias y cuatro optativas, las dos materias restantes soportan créditos de formación básica. Aquí se incluyen materias que intentan incluir todos los conocimientos mencionados en los perfiles en cuanto a su orientación publicitaria, ya sea creatividad, planificación, gestión estratégica o producción.

Desaparecen algunas materias importantes con respecto a planes de estudio anteriores y que en la actualidad están obteniendo importante desarrollo, caso de las industrias culturales que han aparecido y siguen desarrollándose en este entorno digital. No hay alusión alguna a los consumidores o su estudio, incluso cuando se habla del perfil de gestores y estrategas se aplica solo al campo publicitario y se olvida mencionar la importancia que éstos tienen con respecto a la defensa del consumidor (Sánchez Blanco, 2010), a ser sus mayores valedores y no solo actuar de parte del anunciante. 
No hay alusión a los nuevos roles profesionales o los cambios en las estructuras empresariales que se están desarrollando o al estudio y seguimiento de tendencias, un elemento que cada día más importancia están adquiriendo.

a) El módulo de Relaciones Públicas, Comunicación Corporativa e Institucional con un total de 66 créditos ECTS, está constituido por un total de 11 materias, solamente 6 de ellas son obligatorias, 4 optativas y 1 de formación básica. Se incluyen algunas materias de Relaciones Públicas especializadas, materias de propaganda y de comunicación corporativa. La importancia que esta disciplina adquiere en la actualidad ha perdido también un cierto peso en esta titulación, no han desaparecido muchas materias con respecto a planes anteriores pero la mayor parte de las nuevas son asignaturas optativas.

\section{CONCLUSIONES}

Teniendo en cuenta las necesidades o aspectos importantes que planteábamos con respecto a la formación de los futuros profesionales de la comunicación y, teniendo en cuenta los contenidos incluidos en el plan de estudios analizados, nos atrevemos a plantear las siguientes conclusiones:

a) Desequilibrio entre los contenidos aplicados al ámbito de la Publicidad y las Relaciones Públicas, no hay más que ver los créditos de uno y otro módulo (120 y 66) y la categorización de las materias.

b) Si nos detenemos en los cursos de la titulación podemos observar que, por ejemplo, en los dos primeros cursos la diferencia entre los contenidos publicitarios y de relaciones públicas se sitúan en una relación de 7 a 2.

c) Los contenidos que tienen que ver con la creatividad y la elaboración de los mensajes, producción en medios tradicionales y nuevos medios, el estudio de nuevos soportes... solo se plantean desde el punto de vista publicitario cuando cualquiera de estos conocimientos debería aplicarse también al ámbito de las Relaciones Públicas y podían haber constituido materias para ambas disciplinas, lo que habría enriquecido muchos contenidos.

d) Destacaban los profesionales mencionados la importancia del conocimiento del consumidor, sus nuevas relaciones con la tecnología, los medios, sus cambios en actitudes, modos de entender la vida, relacionarse con los demás. No encontramos demasiados contenidos que tenga en cuenta este tipo de cuestiones, salvo una materia en primer curso (Estructura social. consumo $\mathrm{v}$ estilos de vida) aue pensamos debería tener 
e) Creemos que también sería necesarias materias que entre sus contenidos se encontrasen aquellos orientados a enseñar al alumno a buscar información acerca de ese consumidor tan cambiante, conocer tendencias, identificarlas y aplicarlas al margen del medio que se fuese a utilizar.

f) Otro de los elementos destacados se refiere a la importancia de las ideas, en un momento marcado precisamente por ellas y serán ellas también las protagonistas del futuro, sin embargo, no hay materias que tengan en cuenta estos contenidos, las materias sobre creatividad no han aumentado con respecto a planes de estudios anteriores, como ha ocurrido en el caso de otras universidades $\mathrm{y}$, además lo referido a la creatividad se circunscribe a un ámbito muy concreto, como se refleja en el perfil profesional definido como tal.

g) Con respecto a los medios ocurre prácticamente lo mismo que con los contenidos anteriores, se habla de producción publicitaria impresa, audiovisual, web y multimedia (ahora en una misma materia, cuando en el anterior plan de estudios había dos), hay una materia titulada Comunicación alternativa: medios virtuales y nuevos soportes publicitarios y otra titulada Publicidad, artes gráficas y tipografía pero, todo lo referido a medios se asocia a su aplicación publicitaria solamente.

h) En sus descriptores prácticamente todas ellas aluden a la creación de mensajes para esos medios, pero no hay referencia a cómo el individuo los consume o se relaciona con ellos, o cómo han cambiado la configuración del entorno comunicativo y el propio modo de vida de los individuos y sus relaciones.

i) Desaparición de muchos contenidos transversales que podrían relacionar distintos elementos que tienen que ver con todo el proceso comunicativo y los elementos implicados y que no tiene porqué partir necesariamente de él, y que ayudaban a dar una visión más amplia de la titulación dentro del ámbito de las ciencias sociales.

j) En los objetivos específicos de la titulación no existen alusiones a los contenidos mencionados o, por lo menos desde las perspectivas planteadas, lo que significa que no se han tenido demasiado en cuenta los cambios que se están produciendo en el entramado comunicativo. 


\section{REFERENCIAS}

Bravo Navalpotro, J. (2010). Publicidad digital/interactiva: Internet. Revista Telos, 82 de Enero-marzo 2010: 31-42

Carrasco, O. (2009). La idea sigue. Recuperado el 14 de marzo de 2011, de www.marketingnews.es .

Cortés, M (2009). Bienvenido al nuevo marketing. En Del 1.0 al 2.0: claves para entender en nuevo marketing. Impreso por Bubok Publishing. E-Book de acceso gratuito www.clavesdelnuevomarketing.com .

GARCÍA, C (2008). El libro de BOB. Madrid: Nueva Publicidad.

Matatia, I. \& Salzmann, M. (2001). Tendencias. Estilos de vida para un nuevo milenio. Barcelona: Planeta.

Muñoz, F. (2010). Supremacía de las ideas. La comunicación digital, fuente de preocupación. Revista Telos, 82 de enero-marzo: 107-109.

Sánchez Blanco, C. (2010). Aportación de los planificadores estratégicos españoles a la eficacia publicitaria. Revista Latina de Comunicación Social, 65. Recuperado el 20 de diciembre de 2010, de http://www.revistalatinacs.org/10/art2/900_Navarra/22_Cristina.html.

A curiosidade convértese en imprescindible para ser un bo publicitario. (2011). Recuperado el 17 de enero de 2011, de $\underline{\text { www.duvi.es. }}$

Domingo Docampo toma posesión de su segundo mandato como rector de la Universidad de Vigo. (2002). Recuperado el 29 de noviembre de 2010, de www.universia.es

El Anuario del siglo XXI. (2010). Recuperado el 15 de diciembre de 2010, de www.clubdecreativos.com/noticias. 


\section{Montse Vázquez Gestal}

Doctora en Ciencias de la Información por la Universidad Complutense de Madrid. Docente en materias de Creatividad Publicitaria y Estrategias de la Publicidad . Profesora Invitada en universidades mexicanas (Quéretaro, UAM, Cuautemoc), italianas (LUMSA Universitá y La Sapienza), portuguesas (Universidade fernando Pessoa de Porto, Universidade do Minho en Braga). Proyectos de investigación de distintas institucionales autonómicas y nacionales (Xunta de Galicia, Ministerios de Educación y Ciencia, Ministerio de Ciencia e Innovación). 